\title{
Multiple Retroperitoneal Paragangliomas
}

\author{
Santosh Rai $\cdot$ K. Subas $\cdot$ J. Kini $\cdot$ N. Bhat $\cdot$ S. Rao
}

Received: 26 February 2009 / Accepted: 25 April 2012 /Published online: 17 May 2012

(C) Association of Surgeons of India 2012

\begin{abstract}
Paragangliomas are rare with multicentricity being more common in patients with familial history. Early recognition of the characteristic symptoms of headache, palpitations, and diaphoresis in a patient with hypertension and prompt appropriate intervention can minimize the morbidity associated with such tumors and prevent a potentially fatal outcome. Paragangliomas represent neoplasms of neural crest origin that arise from paraganglia. Paragangliomas are tumours arising in extra-adrenal paraganglia either in the paravertebral space. We report a rare case of two discrete paragangliomas in the retroperitoneum of a young girl presenting with throbbing headache. Contrast Enhanced CT Abdomen showed the lesions as a large irregular heterogeneous and intensely enhancing mass lesions. Elevated levels of vanillylmandelic acid were found in the Urine. The lesions were removed by surgery and the histopathologic findings of both the lesions confirmed the diagnosis of multiple paragangliomas. Early identification and early surgery will minimize the potential risks and complications in this condition.
\end{abstract}

Keywords Multiple $\cdot$ Paragangliomas $\cdot$ Retroperitoneal

\author{
S. Rai $\cdot$ K. Subas \\ Department of Radiodiagnosis, KMC Mangalore, \\ Mangalore, India \\ J. Kini \\ Department of Pathology, KMC Mangalore, \\ Mangalore, India \\ N. Bhat $\cdot$ S. Rao \\ Department of Plastic surgery, KMC Mangalore, \\ Mangalore, India \\ S. Rai $(\bowtie)$ \\ Department of Radiodiagnosis, KMC Hospital, \\ Attavar, \\ Mangalore 575001, India \\ e-mail: radiorai@gmail.com
}

\section{Introduction}

Paragangliomas are tumors arising in extra-adrenal paraganglia either in the paravertebral space or more commonly in the paraganglia around the great vessels of the head and neck. They commonly occur singly and sporadically, but may be familial as a part of the multiple endocrine neoplasia syndrome. The lesions that arise from the adrenal medulla are usually designated pheochromocytomas. The lesions that develop in paraganglia other than adrenal medulla are often designated paragangliomas [1]. We report a rare case of multiple discrete paragangliomas in the retroperitoneum of a young girl presenting with throbbing headache.

\section{Case History}

A 14-year-old girl with a history of intermittent throbbing headache with associated fever from 1 month treated with oral medications presented to our hospital. On examination, she was found to be conscious and oriented, but her blood pressure was 134/110 $\mathrm{mmHg}$ (more than 95 percentile). Systolic murmur was heard in the upper left sternal border. She was referred to the department of radiodiagnosis and Imaging for evaluation of hypertension. CT head was unremarkable. Ultrasound of the abdomen showed a heterogeneous lesion measuring around $4 \mathrm{~cm}$ at the lower pole of the left-sided kidney with central areas of necrosis and solid areas. Another lesion measuring around $3 \mathrm{~cm}$ of similar echotexture was noted in the left para-aortic region. A provisional diagnosis of Wilms tumor with regional lymph nodes was furnished. It was thought that Wilms tumor was the cause of hypertension. Contrast-enhanced CT abdomen showed the same lesions as a large irregular heterogeneous and intensely enhancing mass lesion measuring $4.7 \mathrm{~cm} \times$ $4.5 \mathrm{~cm}$ with central area of necrosis (Figs. 1 and 2). Another 


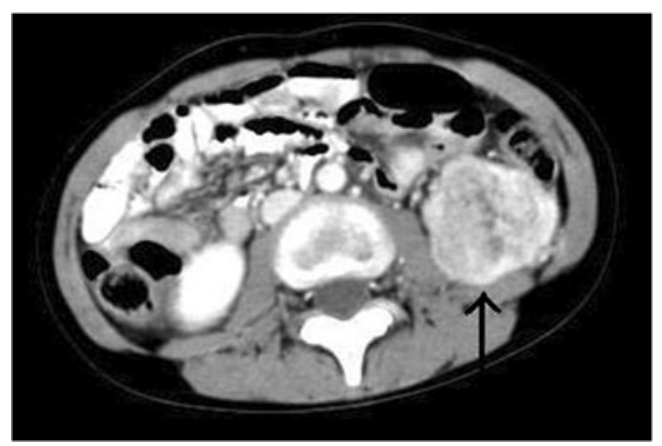

Fig. 1 Contrast-enhanced CT abdomen shows a large irregular heterogeneous and intensely enhancing mass lesion (black arrow) with central area of necrosis inferior to the lower pole of the left kidney in the retroperitoneum

rounded well-defined heterogeneously enhancing mass lesion was found in the left para-aortic region in infrahilar region abutting the abdominal aorta and medial surface of the left-sided kidney (Fig. 3). Subsequently, elevated levels of vanillylmandelic acid were found in the urine. On further probe of the family history, no significant familial history was obtained.

The patient underwent surgery and excision of tumor was done. Intraoperatively, the tumor located in the lower pole of the left-sided kidney was seen to be arising from the capsule of the kidney. Para-aortic mass was also removed. The tumor appeared highly vascular. Intra-operatively blood pressure variations were observed, which was stabilized with medication. Gross specimens appeared solid, homogeneous, and pale brown (Fig. 4). Both the specimens on

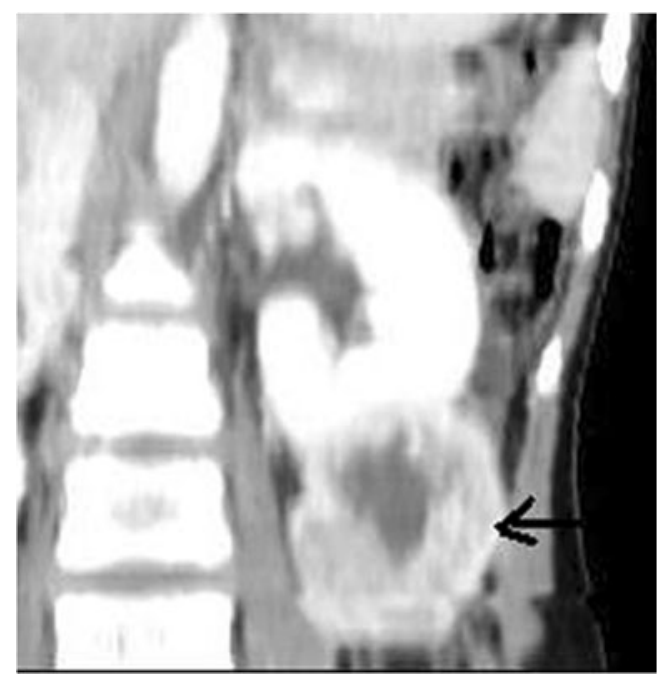

Fig. 2 Coronal reformatted image of the Contrast-enhanced CT abdomen shows a large irregular heterogeneous and intensely enhancing mass lesion (black arrow) with central area of necrosis inferior to the lower pole of the left kidney in the retroperitoneum

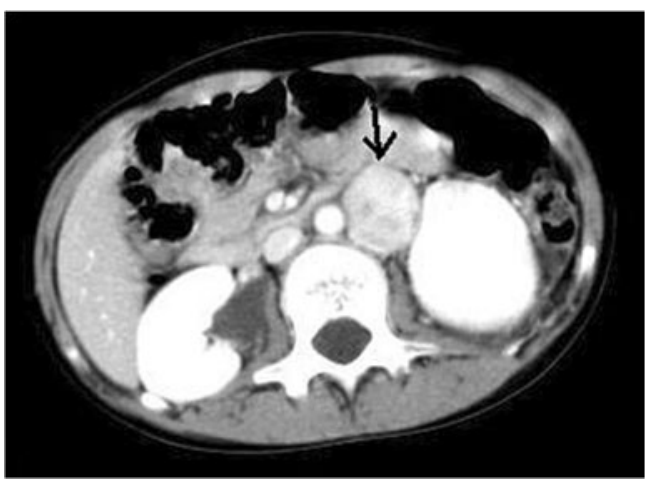

Fig. 3 Rounded well-defined heterogeneously enhancing mass lesion was found in the left para-aortic region abutting the abdominal aorta and medial surface of the left-sided kidney with similar pattern of enhancement (black arrow)

microscopy showed neoplastic cells arranged in an organoid pattern along with anastomosing trabeculae and twisted cell cords. The histopathologic findings of both the lesions confirmed the diagnosis of multiple paragangliomas (Fig. 5). Postsurgery the patient has been doing well and no recurrence has been noted on ultrasound of the abdomen even after 2 years.

\section{Discussion}

Paragangliomas represent neoplasms of neural crest origin that arise from paraganglia. The clinical behavior of paragangliomas is determined by cellular characteristics, secreting capabilities, and tumor location. The symptoms and signs depend on the site of origin and the stage at which it presents [2]. Paragangliomas are rare with multicentricity being more common in patients with familial history. Mutations in the gene encoding succinate dehydrogenase subunit D (SDHD) are responsible for a percentage of hereditary paragangliomas [3].

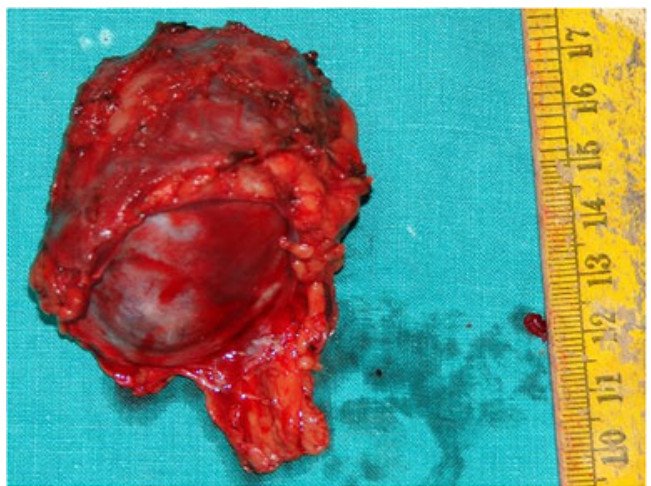

Fig. 4 Intraoperatively the tumours appeared solid, homogeneous, and pale brown 


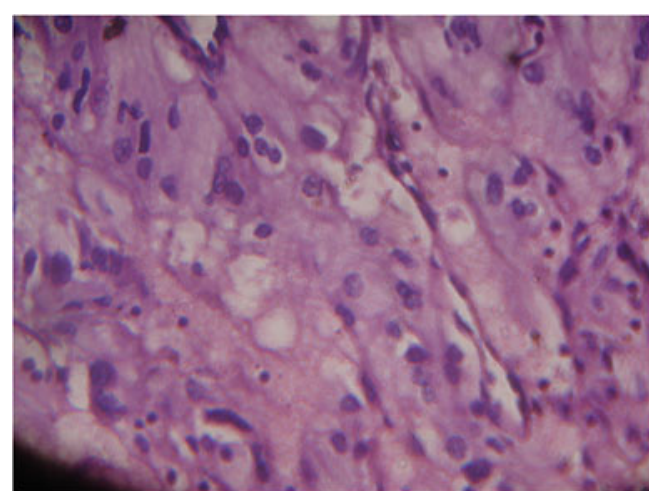

Fig. 5 Microscopy (HE stain) showed neoplastic cells arranged in an organoid pattern along with anastomosing trabeculae and twisted cell cords

Paragangliomas are neuroendocrine tumors, and those occurring in the head and neck have well-recognized familial association. Retroperitoneal paragangliomas are uncommon, and their malignant potential has been very rarely reported in literature [4].

Early recognition of the characteristic symptoms of headache, palpitations, and diaphoresis in a patient with hypertension and prompt, appropriate intervention can minimize the morbidity associated with such tumors and prevent a potentially fatal outcome [5]. Because morbidity after surgical resection increases with tumor size, early surgery will minimize the potential risks.

\section{References}

1. Collins T, Cotran RS, Kumar V, Robbins SL (1986) Robbins Pathologic Basis of Disease, 4th edn. WB Saunders, Philadelphia, pp $1267-1268$

2. Somasundar P, Krouse R, Hostetter R, Vaughan R, Covey T (2000) Paragangliomas - a decade of clinical experience. J Surg Oncol 74 (4):286-290

3. Boedeker CC, Neumann HP, Ridder GJ, Maier W, Shipper J (2005) Paragangliomas in patients with mutations of the SDHD gene. Otolaryngol Head Neck Surg 132(3):467-470

4. Sebastian JP, Williams SE, Wells M, Peake MD (1989) Familial malignant retroperitoneal paraganglioma. Postgrad Med J 65 (768):781-784

5. Petramala L, Cavallaro G, Polistena A, Costesta D, Verrienti A, Ciardi A et al (2008) Multiple catecholamine-secreting paragangliomas: diagnosis after hemorrhagic stroke in a young woman. Endocr Pract 14(3):340-346 